\title{
ARTICLE
}

Clinical Study

\section{MST1/Hippo promoter gene methylation predicts poor survival in patients with malignant pleural mesothelioma in the IFCT-GFPC-0701 MAPS Phase 3 trial}

\author{
Elodie Maille ${ }^{1,2}$, Solenn Brosseau ${ }^{3}$, Vincent Hanoux ${ }^{4}$, Christian Creveuil $^{5}$, Claire Danel $^{6}$, Emmanuel Bergot ${ }^{2,7}$, Arnaud Scherpereel $^{8}$, \\ Julien Mazières ${ }^{9}$, Jacques Margery ${ }^{10}$, Laurent Greillier ${ }^{11,12}$, Clarisse Audigier-Valette ${ }^{13}$, Denis Moro-Sibilot ${ }^{14}$, Olivier Molinier ${ }^{15}$, \\ Romain Corre $^{16}$, Isabelle Monnet ${ }^{17}$, Valérie Gounant ${ }^{18}$, Alexandra Langlais ${ }^{19}$, Franck Morin ${ }^{19}$, Guénaëlle Levallet $\mathbb{D}^{2,20}$ and \\ Gérard Zalcman ${ }^{3,21}$
}

BACKGROUND: The Mesothelioma Avastin Cisplatin Pemetrexed Study (MAPS/NCT00651456) phase 3 trial demonstrated the superiority of bevacizumab plus pemetrexed-cisplatin triplet over chemotherapy alone in 448 malignant pleural mesothelioma (MPM) patients. Here, we evaluated the prognostic role of Hippo pathway gene promoter methylation.

METHODS: Promoter methylations were assayed using methylation-specific polymerase chain reaction in samples from 223 MAPS patients, evaluating their prognostic value for overall survival (OS) and disease-free survival in univariate and multivariate analyses. MST1 inactivation effects on invasion, soft agar growth, apoptosis, proliferation, and YAP/TAZ activation were investigated in human mesothelial cell lines.

RESULTS: STK4 (MST1) gene promoter methylation was detected in 19/223 patients tested (8.5\%), predicting poorer OS in univariate and multivariate analyses (adjusted HR: $1.78,95 \% \mathrm{Cl}(1.09-2.93), p=0.022)$. Internal validation by bootstrap resampling supported this prognostic impact. MST1 inactivation reduced cellular basal apoptotic activity while increasing proliferation, invasion, and soft agar or in suspension growth, resulting in nuclear YAP accumulation, yet TAZ cytoplasmic retention in mesothelial cell lines. YAP silencing decreased invasion of MST1-depleted mesothelial cell lines.

CONCLUSIONS: MST1/hippo kinase expression loss is predictive of poor prognosis in MPM patients, leading to nuclear YAP accumulation and electing YAP as a putative target for therapeutic intervention in human MPM.

British Journal of Cancer (2019) 120:387-397; https://doi.org/10.1038/s41416-019-0379-8

\section{BACKGROUND}

Malignant pleural mesothelioma (MPM) is a rare yet aggressive cancer with poor prognosis mainly caused by occupational asbestos exposure. ${ }^{1}$ Recently, the Mesothelioma Avastin Cisplatin Pemetrexed Study (MAPS) demonstrated the benefit of bevacizumab plus cisplatin/pemetrexed doublet combination on both overall (OS) and progression-free survival (PFS) in 448 MPM patients. $^{2}$ Following this trial, a biological study (Bio-MAPS) is currently evaluating new biomarkers, including the Hippo mammalian sterile 20-like kinase (MST1), as MPM has recently been proven to harbour frequent Hippo pathway alterations. ${ }^{3}$
MST1 (also named STK4), encoded by the human orthologue of the Drosophila melanogaster Hippo gene, and MST2 (STK3) are the best characterised of the five MST kinases (MST1/2/3/4 and YSK1) existing in mammals. MST1/2 primary function is to activate/phosphorylate the large tumour suppressor homologue $1 / 2$ (LATS1/2) kinases, which in turn regulate the Yes-associated protein (YAP) and transcriptional co-activator with PDZ-binding motif (TAZ) activities. Inactive/phosphorylated YAP/TAZ are sequestered in the cytoplasm by proteins such as 14.3 .3 or betacatenin, while active/dephosphorylated YAP/TAZ interact with numerous transcription factors and cause transcription of genes

\footnotetext{
${ }^{1}$ Normandie Université, UNICAEN, INSERM, UMR 1086 ANTICIPE, Caen, France; ${ }^{2}$ Normandie Université, UNICAEN, CEA, CNRS, ISTCT/CERVOxy group, GIP CYCERON, Caen, France; ${ }^{3}$ Department of Thoracic Oncology \& CIC1425, Hôpital Bichat-Claude Bernard, Assistance Publique Hôpitaux de Paris, Université Paris-Diderot, Paris, France; ${ }^{4}$ Normandie Université, UNICAEN, UPRES-EA 2608, Caen, France; ${ }^{5}$ Biomedical Research Unit, CHU de Caen, Caen, France; ${ }^{6}$ Department of Pathology, Hôpital Bichat-Claude Bernard, AP-HP, Université Paris-Diderot, Paris, France; ${ }^{7}$ Department of Pulmonology \& Thoracic Oncology, CHU de Caen, Caen, France; ${ }^{8}$ Department of Pulmonary and Thoracic Oncology, Centre Hospitalier Universitaire Lille, University of Lille, U1019 INSERM, Center of Infection and Immunity of Lille, Lille, France; ${ }^{9}$ Department of Pulmonology, Hôpital Larrey, University Hospital of Toulouse, Toulouse, France; ${ }^{10}$ Medical Oncology Department, Gustave Roussy, Villejuif, France; ${ }^{11}$ Department of Multidisciplinary Oncology and Therapeutic Innovations, Assistance Publique Hôpitaux de Marseille, Aix Marseille University, Marseille, France; ${ }^{12}$ Centre de Recherche en Cancérologie de Marseille (CRCM), INSERM UMR1068, CNRS UMR7258, Aix-Marseille University, UM105 Marseille, France; ${ }^{13}$ Department of Pulmonology, Centre Hospitalier Toulon Sainte-Musse, Toulon, France; ${ }^{14}$ Pôle Thorax et

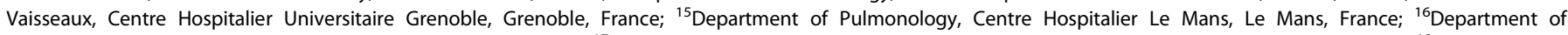
Pulmonology, Pontchaillou University Hospital, Rennes, France; ${ }^{17}$ Department of Pulmonology, Centre Hospitalier Intercommunal Créteil, Créteil, France; ${ }^{18}$ Department of

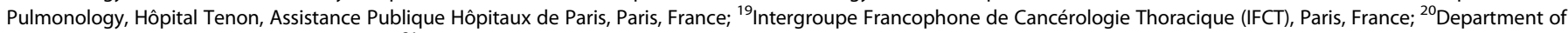
Pathology, CHU de Caen, Caen, France and ${ }^{21}$ U830 INSERM "Genetics and Biology of Cancers, A.R.T group", Curie Institute, Paris, France

Correspondence: Gérard Zalcman (gerard.zalcman@aphp.fr)

Both supervising authors contributed equally: Guénaëlle Levallet, Gérard Zalcman
}

Received: 5 September 2018 Revised: 15 December 2018 Accepted: 20 December 2018

Published online: 11 February 2019 
involved in cell motility, growth, proliferation, and apoptosis. ${ }^{4}$ Thus, MST1 or MST2 loss results in hyperproliferation and tumourigenesis, commonly negated by YAP inactivation. ${ }^{5}$ MST1/ 2 kinases were also reported as contributing to the regulation of (i) apoptosis by establishing a complex with RASSF1A and CNK1 proteins $^{6,7}$ or with the apoptosis-inhibiting protein kinase $\mathrm{CK} 2{ }^{8}$ (ii) cell-cycle progression by catalysing the mitotic phosphorylation of MOBKL1A/1B, ${ }^{9-13}$ and (iii) migration/invasion processes by stabilising lamellipodial F-actin. ${ }^{14-16}$

Several studies suggest that Hippo signalling pathway deregulation is involved in pleural carcinogenesis, since the RASSF1A tumour suppressor gene, an upstream negative regulator of the pathway, is frequently methylated and inactivated in MPM. ${ }^{17,18}$ In our study, we first established a correlation between MST1 kinase gene promoter methylation and reduced OS in MPM patients. By transfecting cells with RNAi-MST1, we further reported that MST1 inactivation increases proliferation, invasion, and cell colony formation of MPM cell lines while decreasing their basal apoptotic activity. Finally, we demonstrated that the effect of MST1 expression loss depends on inappropriate activation of YAP.

\section{METHODS}

Patients from the MAPS trial

From 13 February 2008 to 5 January 2014, 448 patients were randomly assigned to one of two treatments $(223(50 \%)$ to pemetrexed plus cisplatin and bevacizumab and 225 (50\%) to pemetrexed plus cisplatin). Specific informed consent was obtained for the biological studies (Bio-MAPS), and the trial was approved by the appropriate ethics committee (CPP Ref 2007-20 Nord-Ouest III, France).

DNA extraction and methylation-specific PCR assay

DNA samples from MPM were obtained from paraffin-embedded tumour tissue using the QIAamp DNA FFPE Tissue kit (Qiagen). Genomic DNA bisulphite modification was performed using the Epitect kit (Qiagen), according to the manufacturer's instructions and as previously described. ${ }^{19}$ Polymerase chain reaction (PCR) was conducted with specific primers for either the methylated or unmethylated alleles (Table S1) in standard conditions for the following genes encoding proteins of the Hippo pathway or RASSF superfamily: RASSF1A, RASSF2A, RASSF5, RASSF10, MST1 (Figure S1), MST2, LATS1, and LATS2. RASSF6 methylation status was determined by the COBRA technique, as previously described. ${ }^{20}$

Cell culture and transfection

Human MPM cell lines MSTO-211H, NCl-H2452, NCl-H28, and NCl$\mathrm{H} 2452$ from the American Tissue Culture Collection (ATCC) were maintained in RPMI-1640 medium supplemented with $10 \%$ fetal bovine serum, $10 \mathrm{mM}$ L-glutamine and streptavidin/penicillin, and kanamycin $(100 \mu \mathrm{g} / \mathrm{ml})$. Cells were transfected using JetPRIME (Polyplus-transfection ) with small-interfering ribonucleic acid (siRNA) or plasmid DNA, as listed in Table S2.

Reverse transcription-quantitative real-time PCR

After RNA extraction, reverse transcription-quantitative real-time PCR (RT-qPCR) was performed with primer sets (Table S3) as previously described. ${ }^{21}$ RT-qPCR data were normalised to the human glyceraldehyde-3-phosphate dehydrogenase (GAPDH). Relative quantification was calculated using the delta-delta-Ct method.

Immunoblotting

Whole-cell protein extracts were prepared as previously described, ${ }^{22}$ and proteins were detected by immunoblotting with the primary antibody from cell signalling (E-Cadherin, MST1, YAP/TAZ, P-Ser127YAP, GAPDH, vimentin), diluted to 1:1000 in Tween (0.1\%)-tris-buffered saline buffer and horseradish peroxidase-conjugated secondary antibody, and then revealed by enhanced chemiluminescence using the ECL kit (Promega ${ }^{\mathrm{TM}}$ ). Densitometry results of western blot were analysed with ImageJ software. The signal intensity of each band was normalised with GAPDH densitometry values.

Immunofluorescence and image analysis

Transfected cells were fixed and permeabilised as previously described. ${ }^{20}$ The primary antibodies were YAP (Cell Signaling, 1/150), TAZ (Cell Signaling, 1/150), alpha-tubulin (Sigma Aldrich, 1/300), actin (Cell Signaling, 1/300), Fascin (Cell Signaling, 1/300) or cytochrome $C$ (BD Biosciences, 1/50). The AlexaFluo633, AlexaFluo555 or 488-labelled (Invitrogen ${ }^{\mathrm{TM}}$ ) secondary antibodies were added for $1 \mathrm{~h}$. Coverslips were mounted with 4',6-diamidino2-phenylindole (DAPI; Santa $\mathrm{Cruz}^{\mathrm{TM}}$ ) and images captured using high-throughput confocal microscopy (FluoView FV1000, Olympus ${ }^{\mathrm{TM}}$ ).

Quantification and measure of cell cytoplasmic extensions After immunolabeling tubulin filaments, number and length of cytoplasmic extensions from almost 200 cells were assayed from 10 images captured randomly at $20 \times$ objective with a highthroughput confocal microscopy (FluoView FV1000, Olympus ${ }^{\mathrm{TM}}$ ) using ImageJ software (version 1.50d).

\section{Invasion}

A total of $15 \times 10^{3}$ cells were added in serum-free medium to the top invasion chambers of 24-well transwell plates containing cell culture insert with $8 \mu \mathrm{m}$ pores (BD BioCoat Matrigel ${ }^{\oplus}$ Invasion Chamber, BD Biosciences $^{\mathrm{TM}}$ ). Complete media supplemented with hepatocyte growth factor were added to the bottom chambers. Cells were incubated for $48 \mathrm{~h}$ and then removed; migrating (bottom) cells were stained with crystal violet.

\section{Soft agar assay}

Base agar matrix $(100 \mu l$, Cell Biolabs) was seeded in a 96-well plate and 1500 cells/well layered on agar followed by $50 \mu \mathrm{l}$ of $2 \times$ complete medium. After 25 days, colonies were stained and counted for each well.

Spheroid culture

At $24 \mathrm{~h}$ post transfection, cells were reseeded with complete medium in 24-well plates without adhesion (Nunclon ${ }^{\mathrm{TM}}$ Sphera $^{\mathrm{TM}}$ Microplates, Thermo Fisher Scientific). Sphere formation was evaluated on day 6 at $\times 10$ magnification with a phase-contrast inverted microscope.

\section{Apoptosis measurement}

DNA fragmentation and Caspase $3 / 7$ activation were assayed using the Cell Death Detection ELISA plus kit (Roche, USA) and the Caspase-Glo 3/7 Luminescence Assay (Promega Corp. Madison, WI, USA), respectively, according to the manufacturer's instructions.

Bromodeoxyuridine incorporation

Cells were transfected, left for $24 \mathrm{~h}$, then labelled with bromodeoxyuridine (BrdU) (1:500 dilution, cell proliferation assay, Millipore) for a further 24 or $48 \mathrm{~h}$. BrdU incorporation was measured according to the manufacturer's instructions.

\section{Statistical analysis}

The Bio-MAPS study was a pre-planned ancillary and exploratory study. The characteristics of patients with promoter methylation analysis were compared to those without using chi-squared tests or Fisher's exact tests for qualitative variables, and Student's $t$-tests or Mann-Whitney tests for quantitative variables.

The prognosis value of MST1 promoter hypermethylation was assessed for PFS and OS using univariate and multivariate Cox models. Cofactors introduced to the multivariate model were: 
treatment arm, stratification factors used in the MAPS trial (histology, performance status (PS), and smoking status), and clinical factors known to be associated with survival (gender, age, sex, haemoglobin, white blood cells, and platelets).

Hazard ratios (HRs) were estimated with their 95\% confidence intervals $(95 \% \mathrm{Cls})$. Bootstrap resampling was used to assess the model's stability and the optimism-corrected concordance index (cindex). The data were analysed with IBM SPSS software Version 22.0.

In vitro data are presented as means \pm SEM; each experiment was performed at least three times independently. Statistical differences were determined either by one-way analysis of variance (ANOVA) or Dunnett's multiple comparison test to compare each experiment's condition with siMST1 (GraphPad Software, Inc. USA). Statistical significance was set at $p \leq 0.05$.

\section{RESULTS}

MST1 promoter hypermethylation predicts shorter OS of MPM patients

Assessing the baseline characteristics of patients from the MAPS trial reveals that biological parameters (haemoglobin, platelet count, leucocyte count), general status (PS $0-1$ versus $>2$ ), and histology (epithelioid vs. sarcomatoid/mixed) are potent prognostic factors of OS $(p<0.05)$ and PFS in univariate and multivariate analyses (2) in this subset of MAPS patients. The baseline characteristics of the 223 patients in whom gene methylation assays were performed did not differ from those of patients for whom such analyses were not possible (Table S4). RASSF1A, RASSF2A, RASSF6, and RASSF10 were found to be methylated in $11.1 \%, 14.5 \%, 21.5 \%$, and $4.4 \%$ of samples, respectively, while no sample exhibited any RASSF5, MST2, LATS1, or LATS2 methylation. None of these methylations influenced survival in univariate analysis (data not shown).

MST1 promoter methylation status was available for 223/448 patients from MAPS (Fig. 1a), and MST1 promoter was methylated in 19/223 samples (8.5\%) (representative methylation-specific PCR (MS-PCR) are shown in Figure S1).

In univariate analysis, the median OS of patients with methylated MST1 promoter was 1.4 times lower than that of patients with unmethylated MST1 promoter (13.0 versus 19.3 months, HR: $2.40,95 \% \mathrm{Cl}(1.48-3.90), p<0.001)$ (Fig. 1b). Moreover, univariate analyses showed that methylation of MST1 gene predicted worse survival in each treatment group separately, with $\mathrm{HR}=2.29(95 \% \mathrm{Cl} 1.13-4.65), p=0.021$ for the chemotherapy arm and 2.50 (95\% Cl 1.27-4.90), $p=0.0079$ for the bevacizumab arm (Figure S2).

This result was confirmed in multivariate analysis in the whole series, both treatment arms analysed together (adjusted HR: 1.78, $95 \% \mathrm{Cl}(1.09-2.93), p=0.022$, adjustment for gender, age, histology, PS, haemoglobin, leucocyte and platelet counts, smoking, and treatment arm) (Fig. 1b), and validated by a bootstrap procedure: MST1 methylation was significantly associated with worse OS in 59\% of 1000 bootstrapped samples, with an optimism corrected c-index of 0.67 .

When the multivariate analysis was performed separately in the two treatment arms, the survival impact only remained significant in the bevacizumab arm (HR: 2.37, (95\% Cl (1.16-4.83), $p=0.017)$. However, an adjusted interaction test did not support MST gene methylation predictive value, with adj. HR: 1.44 (95\% Cl 0.71-2.93), $p=0.38$ (Figure S2).

Finally, MST1 inactivation did not significantly predict PFS for MPM patients, although there was a correlating trend of a deleterious impact (HR: $1.53,95 \% \mathrm{Cl}(0.95-2.46), p=0.082$ in univariate analysis).

MST1 depletion modifies human mesothelial cell stretching Since MST1 methylation status was the only alteration found to significantly influence prognosis in our large series of MPM a

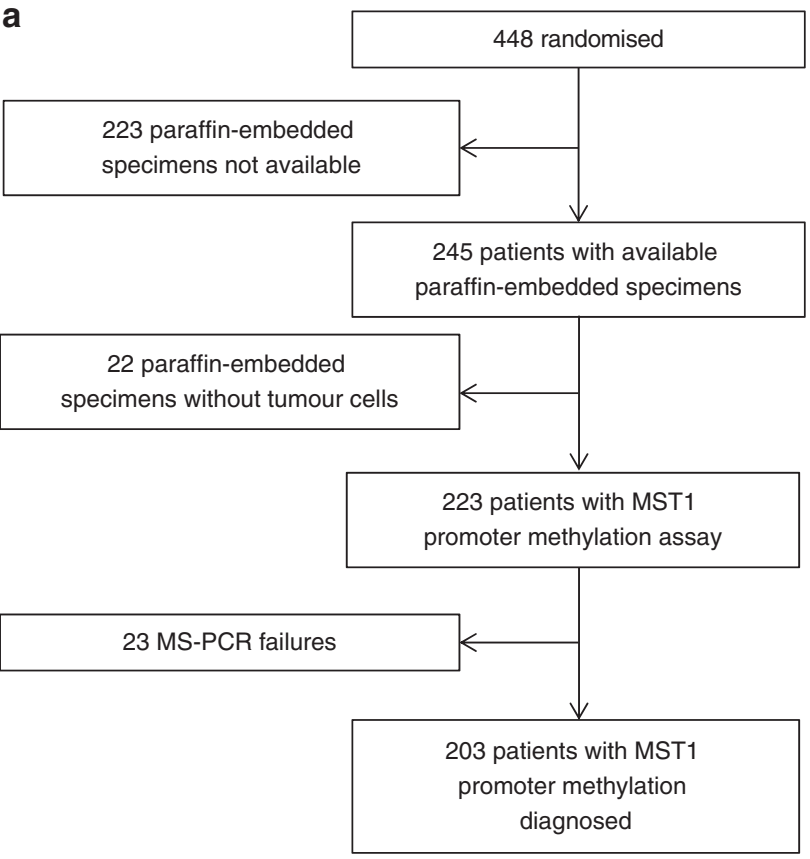

b

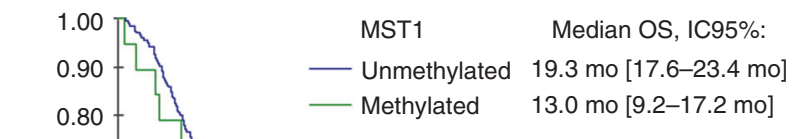

$$
\begin{aligned}
& \text { Number } \quad \begin{array}{llll}
19 & 13 & 4 & 0
\end{array} \\
& \begin{array}{llllllllll}
\text { at risk } & 204 & 157 & 98 & 59 & 35 & 21 & 12 & 7 & 5
\end{array} \\
& \mathrm{HR}=2.40[1.48-3.90], p<0.001 \\
& \text { Adj } \mathrm{HR}=1.78 \text { [1.09-2.93], } p=0.022 \\
& \text { (Adjustment for sex, age, histology, PS, haemoglobin, white } \\
& \text { blood cells, platelet, tabaco and arms) }
\end{aligned}
$$

Fig. 1 a Flow chart. b Kaplan-Meier curve of overall survival according to the MST1 promoter status (methylated or unmethylated)

patients, we focussed our functional mesothelial cell studies on this protein's role.

MST1 was silenced in four human mesothelial cell lines (MSTO$211 \mathrm{H}, \mathrm{H} 2452, \mathrm{H} 28$, and $\mathrm{H} 2452$ ) with normal basal MST1 expression (as evidenced by MS-PCR and qRT-PCR, data not shown), using siRNA-MST1. After testing several small interfering RNA (siRNA) sequences, we retained two that decreased both MST1 mRNA and protein expression by at least $40 \%$ in MPM cells (Fig. 2a, b, S3A). To validate the specificity of the effects observed in the absence of MST1, we have also introduced an experimental condition in which the cells are transfected with both a siMST1 and a plasmid carrying the coding sequence of MST1. The plasmid was brought at a concentration determined to overcome the amount of siRNA silencing the endogenous MST1 (Fig. 2a, b, S3A). 


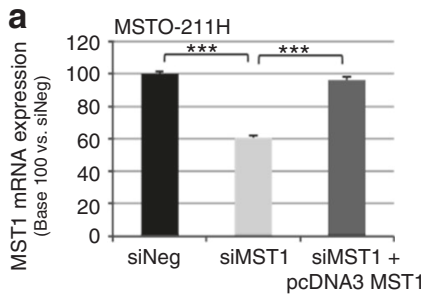

b
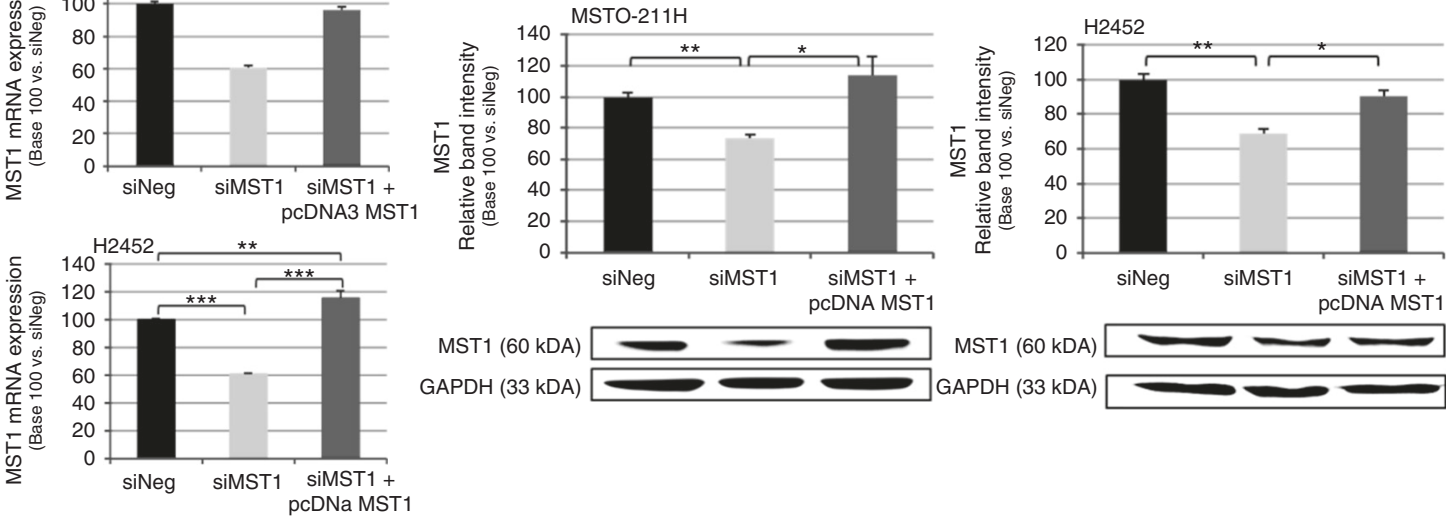

C
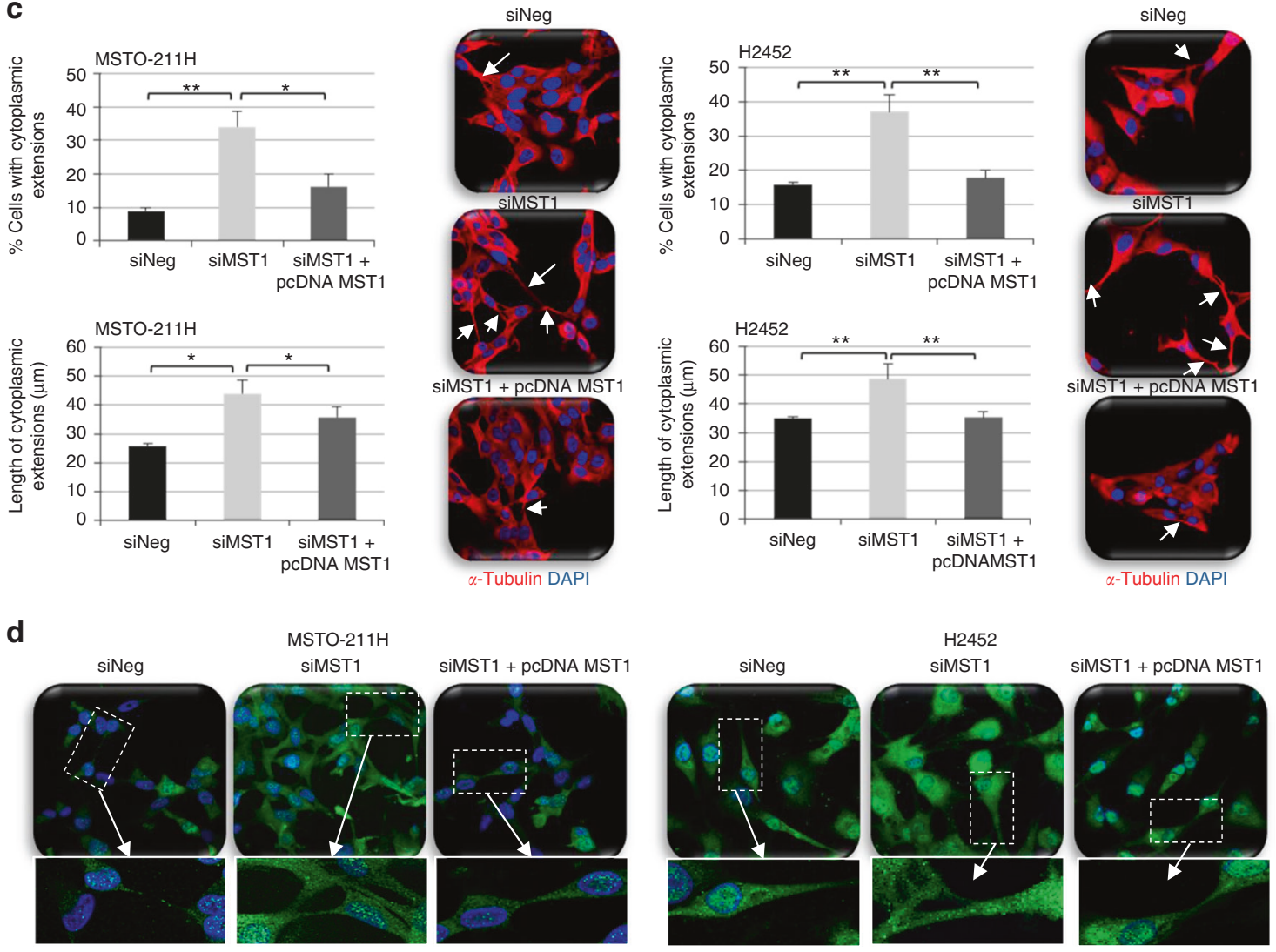

Fascin DAP
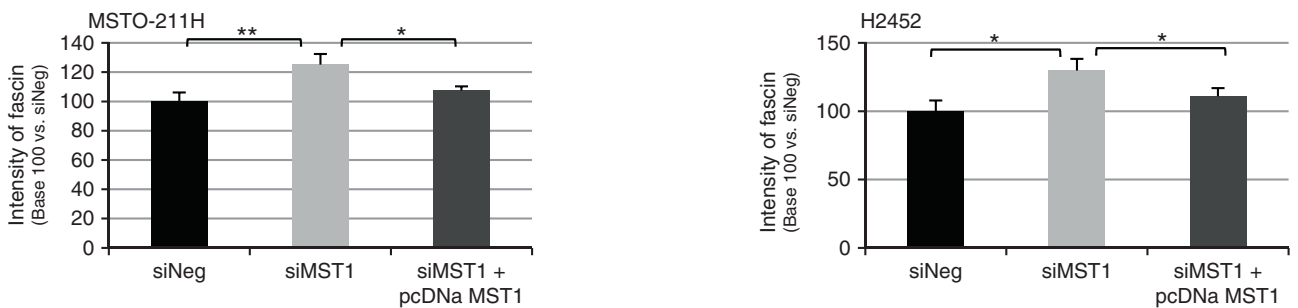

Fig. 2 MST1 depletion causes morphological changes. a-d MST0-211H and H2452 cells were transfected with siNeg, siMST1, or siMST1+pcDNAMST1, analysed $48 \mathrm{~h}$ after transfection. Expression of MST1 was analysed by reverse transcription-quantitative real-time PCR (RT-qPCR) (a) and western blot (b), using glyceraldehyde-3-phosphate dehydrogenase (GAPDH) as an internal control. Quantification of number of cytoplasmic extensions and their size $(\mu \mathrm{m})$ after $\alpha$-Tubulin staining (c) and quantification of Fascin expression (d) by immunofluorescence and confocal microscopy in almost 200 cells using ImageJ software. Representative confocal pictures (c, d) are presented for cells stained for $\alpha$-Tubulin (red), Fascin (green), and nuclei stained with 4',6-diamidino-2-phenylindole (DAPI). For all histograms, error bars indicate the SEM of at least three independent experiments; ${ }^{*} p<0.05,{ }^{* *} p<0.01$, and ${ }^{* * *} p<0.001$, using an analysis of variance (ANOVA) test followed by Dunnett's test 
As evidenced by alpha-tubulin immunostaining, MST1-depleted cells were much more "stretched" than cells transfected with a control siRNA (siNeg). The shape of MSTO-211H or H2452 cells (Fig. 2c) (as $\mathrm{H} 2052$ and $\mathrm{H} 28$, data not shown) appeared significantly modified, and MST1 knockdown cells exhibited more cytoplasmic extensions than mock-transfected cells. Epithelial (E-cadherin) and mesenchymal (vimentin) marker quantification revealed that the morphological changes induced by MST1 loss were not caused by an epithelial-mesenchymal transition, since we found no typical expression switch between epithelial and mesenchymal markers (data not shown).

Cytoplasmic expansions from MST1-depleted cells were shown to be actual filopodia, as confirmed by the fascin enrichment in these structures (Fig. 2d), thus suggesting an increased ability for cell migration upon MST1 knockdown.

MST1 loss increases invasion and growth in agar of MPM cells We observed that MST1 depletion significantly increased the ability of MSTO-211H, H2452 (Fig. 3a), H28, and H2052 (Figure S3B) cells to invade Matrigel ${ }^{\oplus}$. Moreover, colony formation on soft agar was increased by MST1 depletion in MSTO-211H, H2452 (Fig. 3b), $\mathrm{H} 28$, and H2052 (Figure S3C).

Finally, by impeding substrate-cell attachment, we caused spheroid formation increase in MSTO-211H and H2452 cells in absence of the MST1 kinase (Fig. 3c). For MST1-depleted MSTO$211 \mathrm{H}$ cells, we also observed that the cells were more individualised than control cells, which necessarily group into cell clumps.

MST1 depletion decreases basal apoptosis and increases proliferation of MPM cells

MSTO-211H and $\mathrm{H} 2452$ cell lines transfected with siMST1 presented significantly reduced DNA fragmentation (Fig. 4a), suggesting decreased apoptosis. This result was consistent with the decreased caspase 3/7 activity also measured in the absence of MST1 in the MSTO-211 H and $\mathrm{H} 2452$ cells (Fig. 4b), as in $\mathrm{H} 28$ and H2052 cells (Figure S3D), and the lower intensity of cytochrome $C$ staining quantified in MSTO-211H and H2452 cells (Fig. 4c), all assays exploring apoptosis ability.

This basal apoptotic activity decrease in MST1-depleted cells was accompanied by increased cell proliferation for MSTO-211H and $\mathrm{H} 2452$ cells (Fig. 4d), or H28 and H2052 cells (Figure S3E), as measured by BrDU incorporation.

MST1 loss leads to nuclear YAP accumulation but TAZ cytoplasmic retention

MST kinases are known to activate LATS kinases, ${ }^{4}$ which in turn are able to phosphorylate YAP, inducing either YAP cytoplasmic sequestration or YAP proteasome degradation. ${ }^{4}$ It should be noted that in MSTO- $211 \mathrm{H}$ cells, cells with LATS1 kinase inactivation, ${ }^{23}$ the Hippo pathway remains functional as demonstrated by the increase of phosphorylation on the serine 127 of YAP with increasing cell density (Figure S4A) and the concomitant decrease in intensity of nuclear YAP (Figure S4B), LATS1 inactivation being probably compensated by another NDR kinases.

By using immunocytochemistry, we actually found that MST1 depletion led to nuclear YAP accumulation, suggesting a decrease in YAP phosphorylation. Conversely, using specific TAZ antibodies, we observed a decrease in nuclear TAZ, a YAP close homologue, in MSTO-211H and $\mathrm{H} 2452$ mesothelial cells (Fig. 5a).

We evaluated YAP/TAZ transcriptional activity by quantifying the expression of YAP/TAZ target genes: CTGF, ANKDR1, and Cyr61. CTGF expression did not significantly vary upon MST1 depletion (data not shown), while ANKDR1 and Cyr61 expression actually significantly increased by $1.6-$ to 2.5 -fold, as shown in $\mathrm{H} 2452$ (Fig. 5b) and H2052 cells (Fig. 5c).

Western blot analyses further confirmed that YAP protein was more expressed in MST1-depleted MPM cell lines, while TAZ protein was less expressed. Furthermore, YAP protein should accumulate under its active form as evidenced by the P-Ser127YAP decrease detected in these cells, this form being reputed to be inactive (Fig. $5 \mathrm{~d}$ ).

We next evaluated apoptosis activity, invasion, and colony formation of MPM cell lines in the absence of either YAP or TAZ (Fig. 6, Figure S5). We found that YAP and TAZ extinction actually decreased MPM cell invasion (Fig. 6a, Figure S5,A) and colony numbers (Fig. 6b, Figure S5,B), and increased caspase $3 / 7$ activity (Fig. $6 c$, Figure $S 5, C$ ). Thus, the aggressive cell phenotype induced by MST1 loss could result from YAP cytoplasmic-to-nuclear shuttle and TAZ cytoplasmic retention. By silencing YAP in MST1-depleted MSTO-211H or H2452 cells, in line with such hypothesis, we were finally able to decrease their invasion through Matrigel ${ }^{\infty}$ (Fig. $6 \mathrm{~d}$ ).

\section{DISCUSSION}

Today, there is still no oncogenic "driver" identified in MPM enabling targeted therapeutics to be developed. ${ }^{24}$ To identify such a putative driver, the Bio-MAPS study set out to characterise the molecular abnormalities in tumours from patients enroled in the MAPS phase 3 trial. Focusing on Hippo pathway alterations, we assayed MST1 gene promoter hypermethylation, for the first time to our best knowledge, in a subset (8.5\%) of MPM patients. MST1 promoter hypermethylation had been previously described in not only other cancers such as sarcoma ${ }^{18}$ and head and neck squamous cell carcinomas, ${ }^{25}$ but also in non-cancer diseases like autoimmune pancreatitis and rheumatoid arthritis. ${ }^{26}$ While MST1 inactivation had not previously been evidenced in MPM, our findings are in line with the reports of frequent genetic alterations of Hippo pathway members recently revealed in $\mathrm{MPM}^{23}$ and support the concept that Hippo pathway alterations are key events in pleural carcinogenesis. ${ }^{23,27,28}$

Interestingly, our study further revealed that the hypermethylation of MST1 promoter is associated with significantly worse OS for MPM patients, which is consistent with its tumour suppressor function and biomarker potential status. Our findings are consistent with those already reported in the literature for other cancers, such as breast cancer, ${ }^{29}$ hepatocellular carcinoma, ${ }^{30}$ and colorectal cancer. ${ }^{31}$

By mimicking MST1 loss in mesothelioma cell lines via MST1 RNA interference (RNAi) transfection, we demonstrated that MST1 prevented nuclear YAP accumulation while permitting TAZ cytoplasmic retention in mesothelial cells, thus inhibiting cell motility, growth without anchorage, and proliferation, while controlling basal apoptosis. These results are consistent with data from the literature, where MST1 role in invasion, migration, apoptosis, and cell proliferation has already been documented in various cancer models, ${ }^{15,16,32-35}$ though not yet in MPM. Moreover, MST1 or MST2 loss is known to lead to hyperproliferation and tumourigenesis, which are commonly prevented by concurrent YAP inactivation. ${ }^{5}$ Conversely, MST1 overexpression induces apoptosis, inhibits proliferation and tumour growth, and leads to YAP phosphorylation on Ser127 (thus YAP inactivation), subsequently provoking CTGF, AREG, and survivin (YAP-target genes) downregulation in hepatocellular carcinoma $^{36}$ and non-small cell lung cancer. ${ }^{37}$ In line with such reports, our study found that the nuclear YAP accumulation induced by MST1 depletion is consistent with a P-Ser127YAP decrease in MST1-depleted cells with ANKDR1 and Cyr61 gene transcription increase, supporting the fact that YAP accumulated under its active form in the nucleus. We also revealed that YAP inactivation in MST1-depleted cells was able to fully reverse their ability to invade Matrigel ${ }^{\oplus}$. The increase in nuclear YAP in the absence of MST1 could explain the poorer OS in patients with MPM and the more "aggressive" phenotype of mesothelial cells at the cellular level. That loss of MST1 expression involving YAP has already been reported in the literature, ${ }^{34}$ as has the involvement of YAP in the tumour progression of MPM, yet our 
a
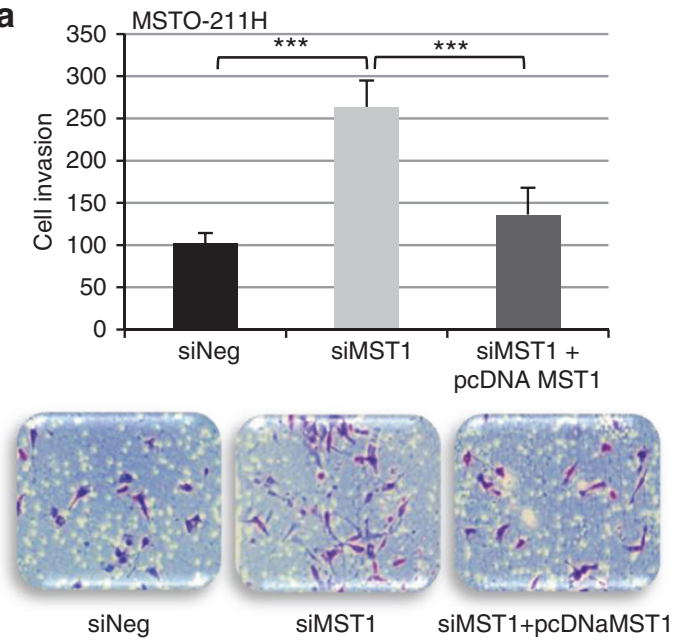

b
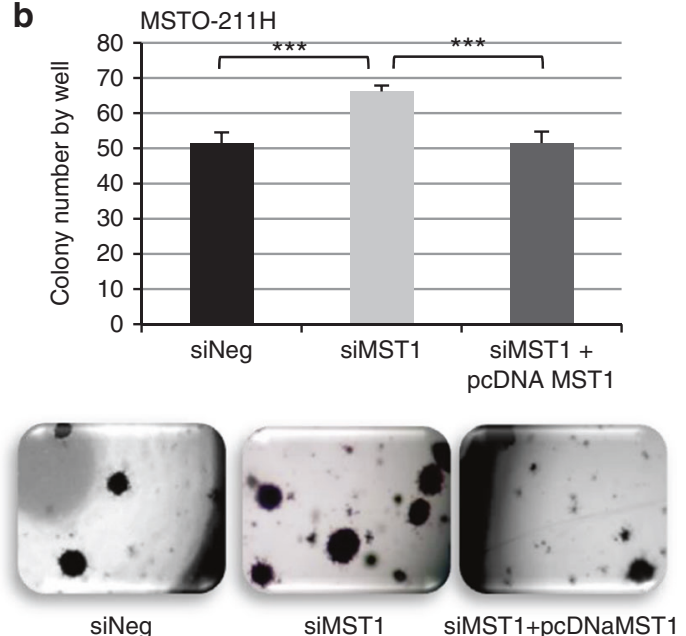

C

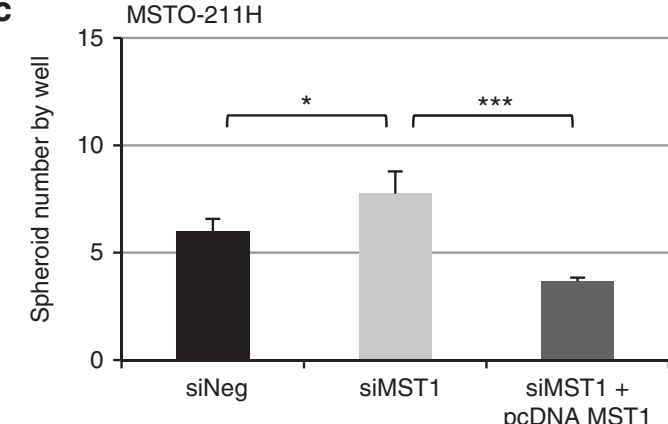

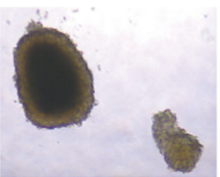

siNeg

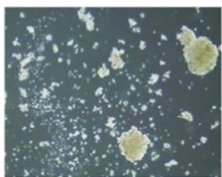

siMST1

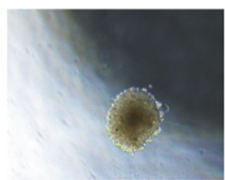

siMST1+pcDNaMST1
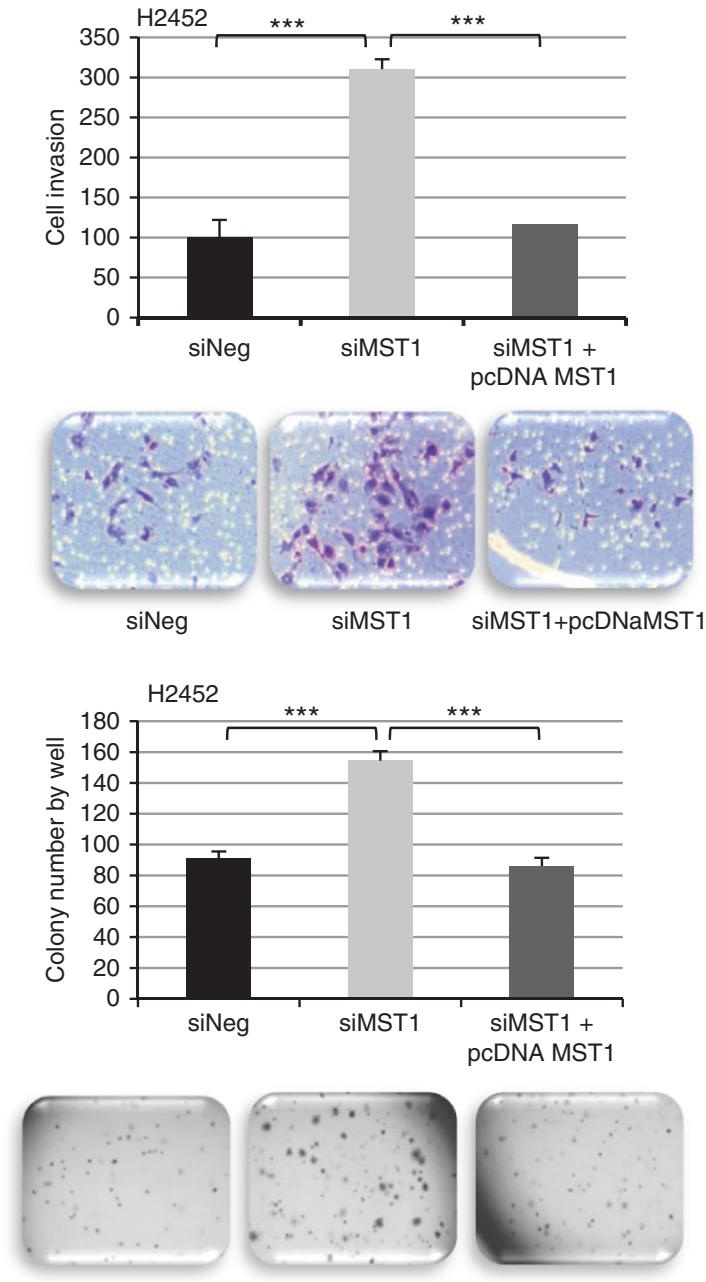

siNeg

siMST1

siMST1+pcDNaMST1

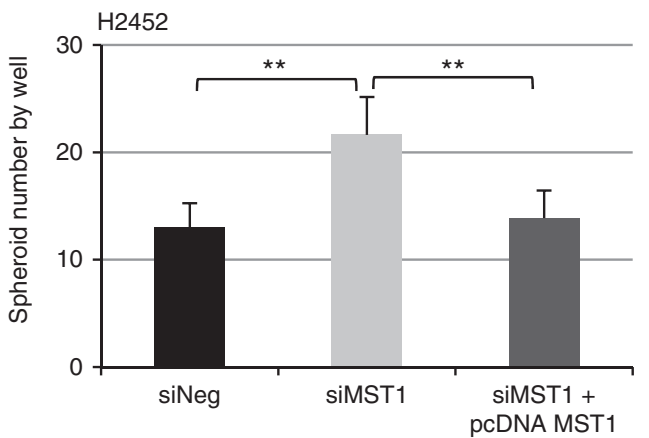

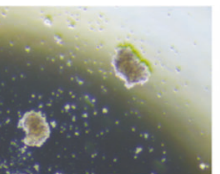

siNeg

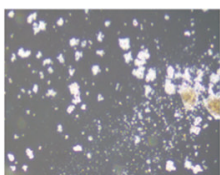

siMST1

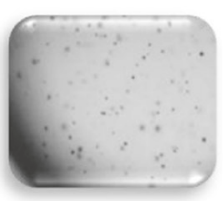

Fig. 3 MST1 depletion increases cell invasion and anchorage-independent growth and decreases spheroid diameter. a-c MST0-211H and H2452 cells were transfected with siNeg, siMST1, or siMST1+pcDNAMST1, experiments were performed $48 \mathrm{~h}$ after transfection. a Invasion capacity of transfected cells on BioCoat Matrigel Invasion Chamber for $48 \mathrm{~h}$. b Quantification of colonies in soft agar after 21 days. c Quantification of spheroid after 6 days. Representative picture is provided under histograms. For all histograms, error bars indicate the SEM of at least four independent experiments; ${ }^{*} p<0.05,{ }^{* *} p<0.01$, and ${ }^{* *} p<0.001$, using an analysis of variance (ANOVA) test followed by Dunnett's test 

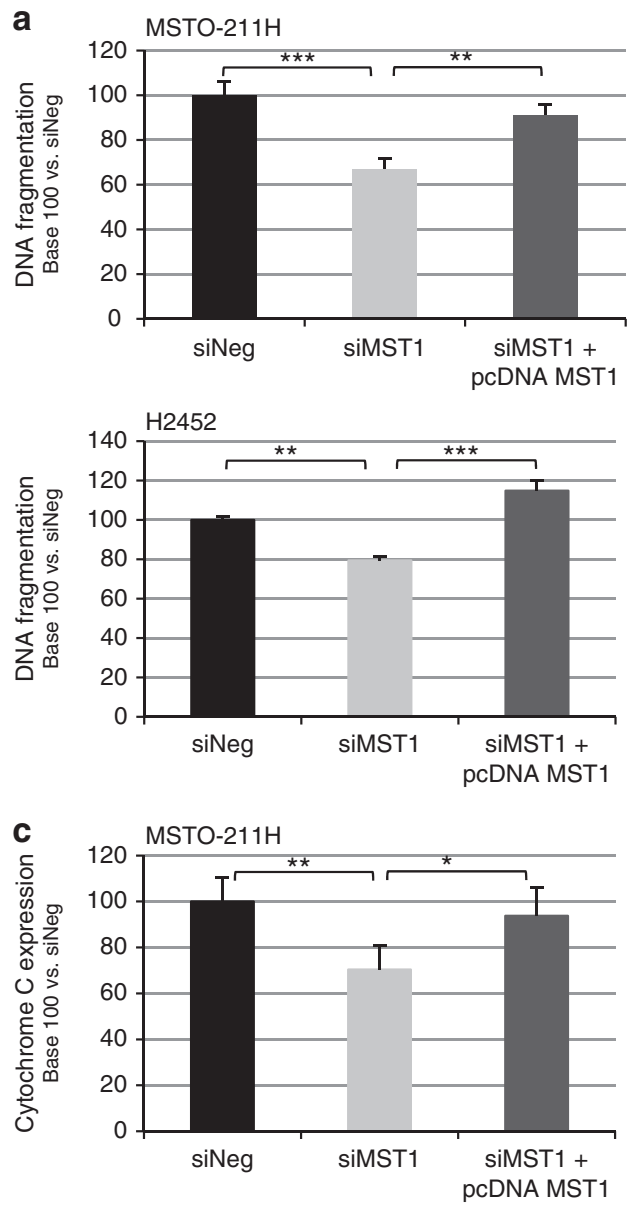

Cytochrome C DAPI

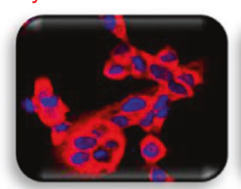

siNeg

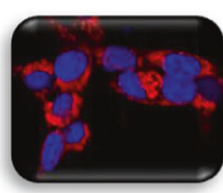

siMST1

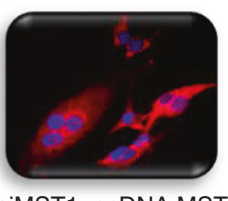

siMST1+pcDNA MST1 b
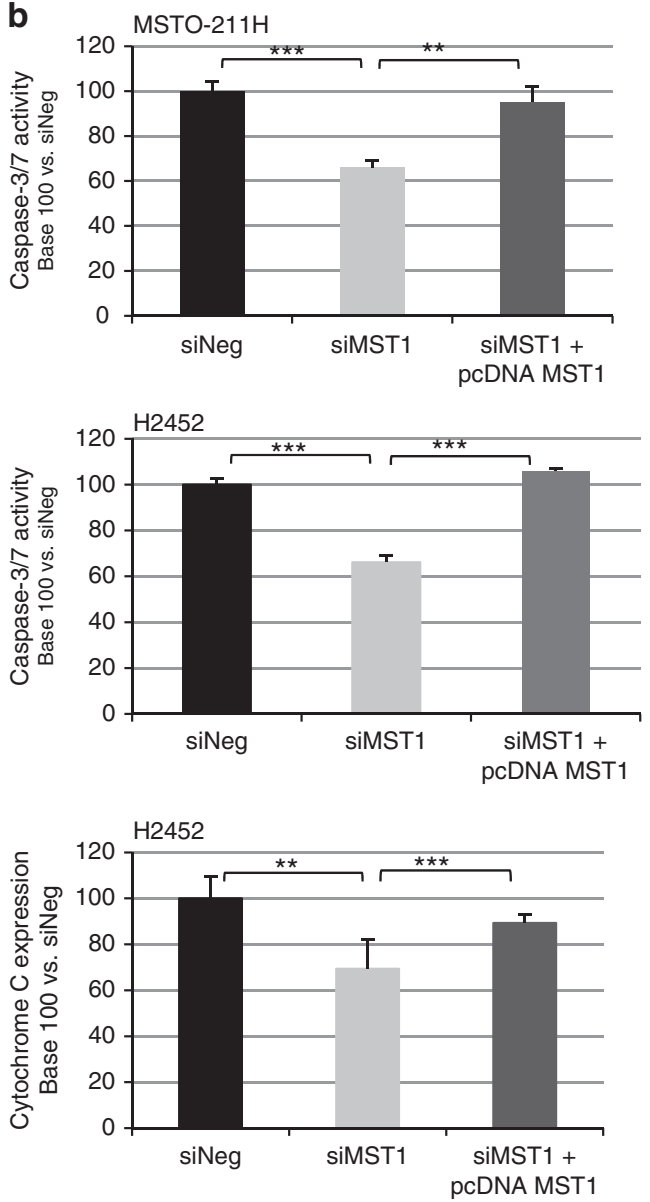

Cytochrome C DAPI

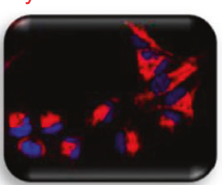

siNeg

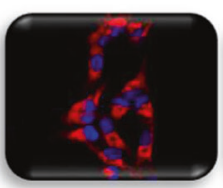

siMST1

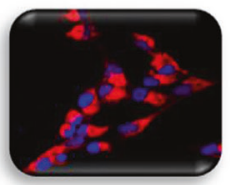

siMST1+pcDNA MST1 d
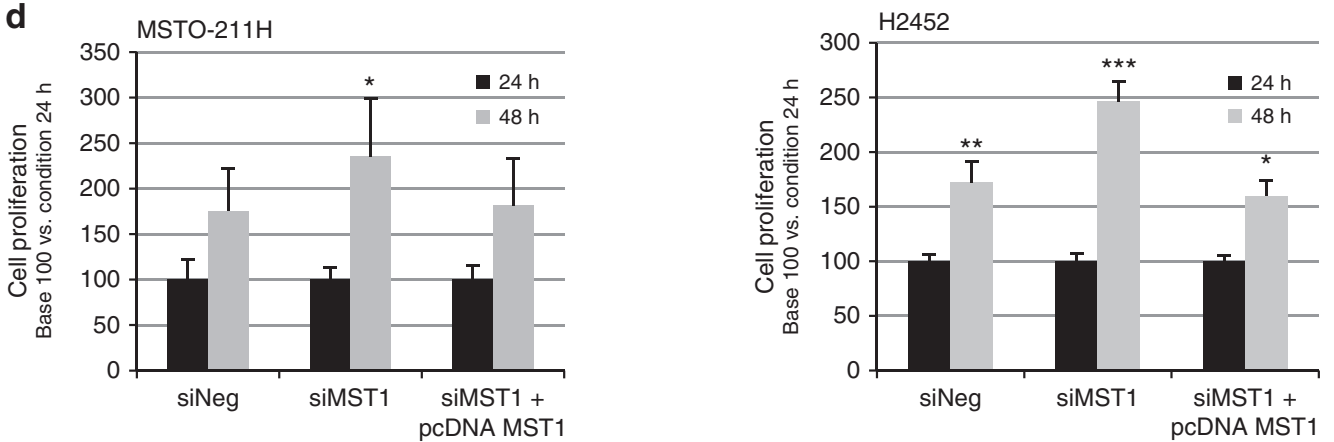

Fig. 4 MST1 depletion decreases apoptosis and increases cell proliferation. a-d MST0-211H and H2452 cells were transfected with siNeg, siMST1, or siMST1+pCDNAMST1. Experiments performed $48 \mathrm{~h}$ after transfection. a Caspase 3/7 activity. b DNA fragmentation. c Quantification of cytochrome $c$ (red) and representative immunofluorescent confocal picture, nuclei stained with 4',6-diamidino-2-phenylindole (DAPI). d Cell proliferation assays. For all histograms, error bars indicate the SEM of at least three independent experiments; ${ }^{*} p<0.05$, ${ }^{* *} p<0.01$, and ${ }^{* * *} p<$ 0.001 , using an analysis of variance (ANOVA) test followed by Dunnett's test

study was the first to identify Hippo kinase inactivation leading to YAP deregulation.

YAP has been evidenced as a potential therapeutic target in MPM patients. ${ }^{25,38,39}$ In line with our results, another team recently reported that YAP inactivation in the MSTO- $211 \mathrm{H}$ cells, which contain an inactive kinase-truncated LATS1 fusion protein and thus a constitutively nuclear active YAP by wild-type LATS1 kinase overexpression leading to YAP phosphorylation, was able to restore YAP to its cytoplasmic location, consisting of a surrogate marker for its functional inactivation . In such YAP-inactivated 
a
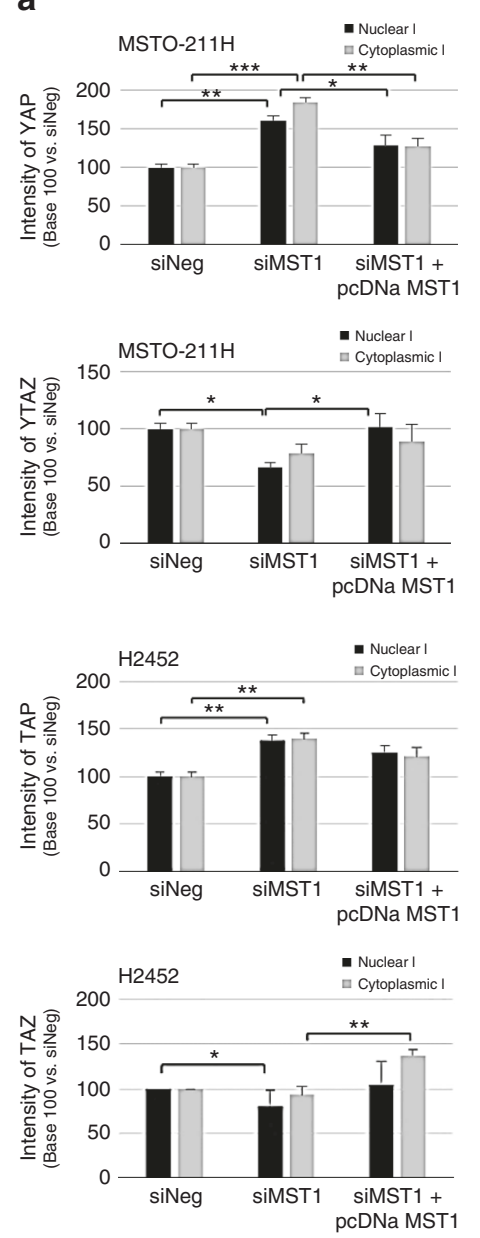

b
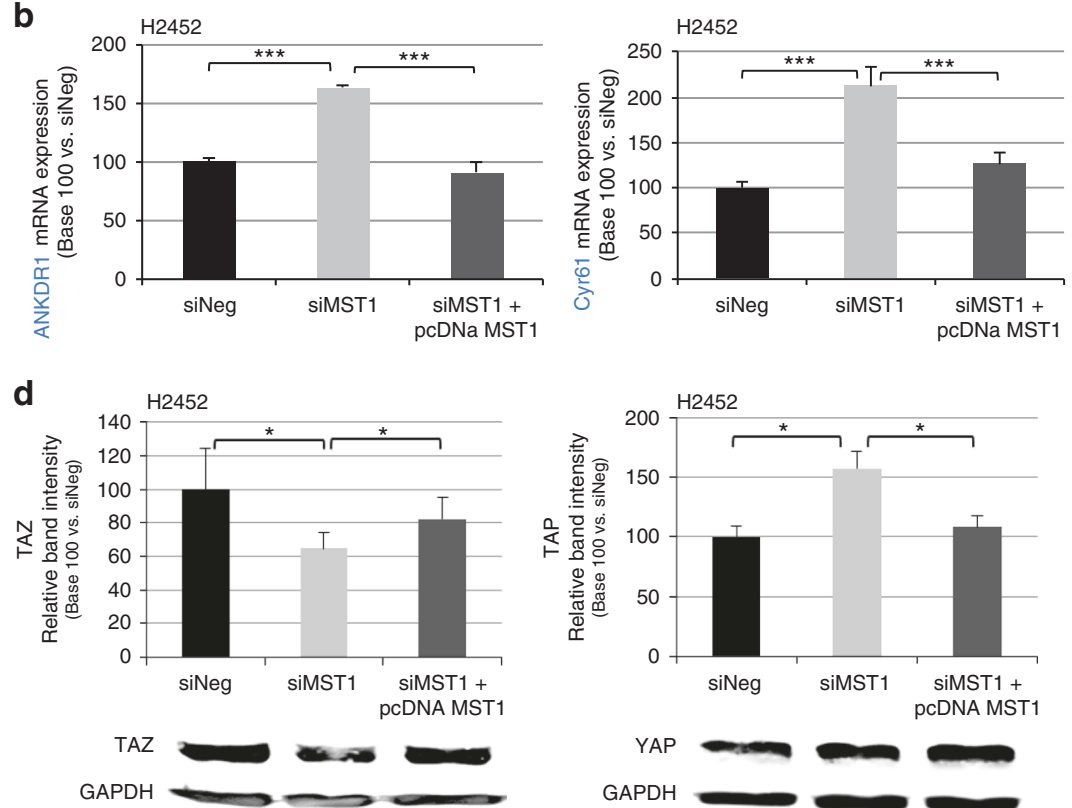

MSTO-211H:
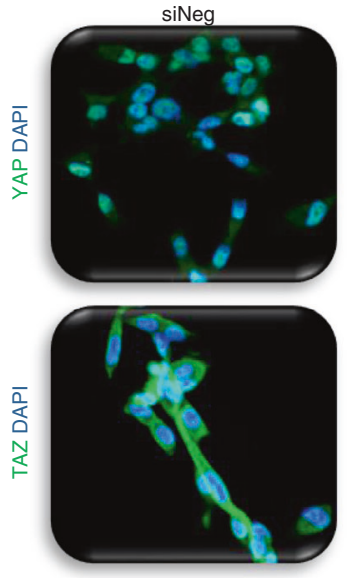

H2452:
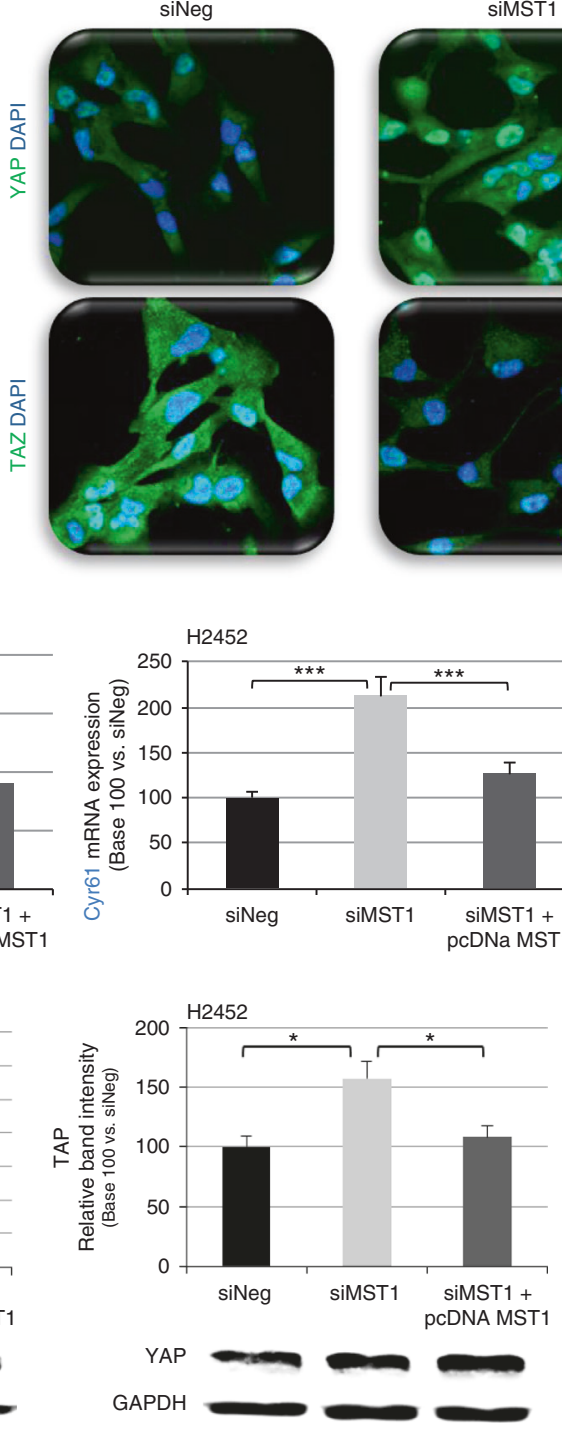
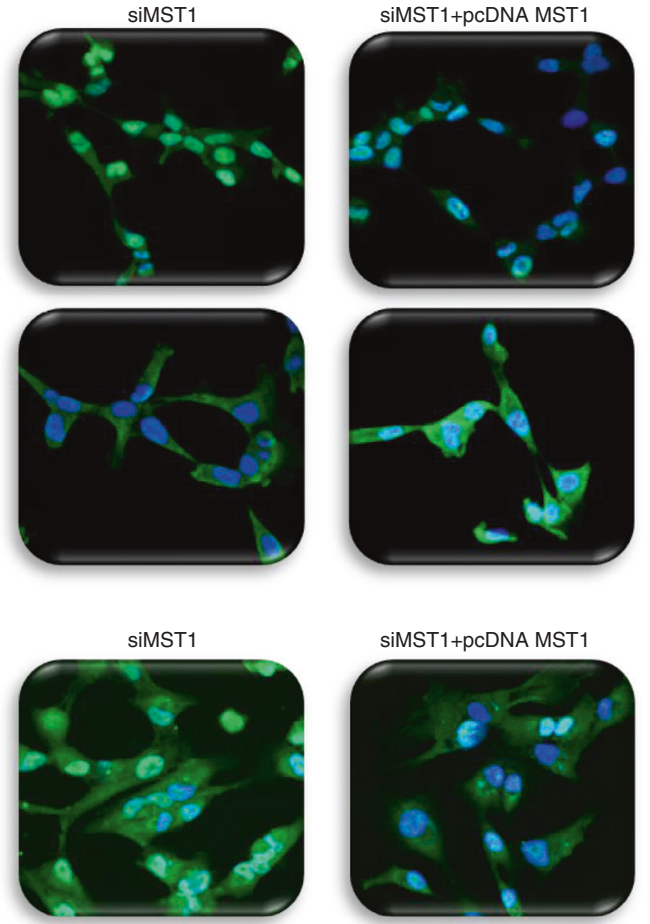

siMST1+pcDNA MST1
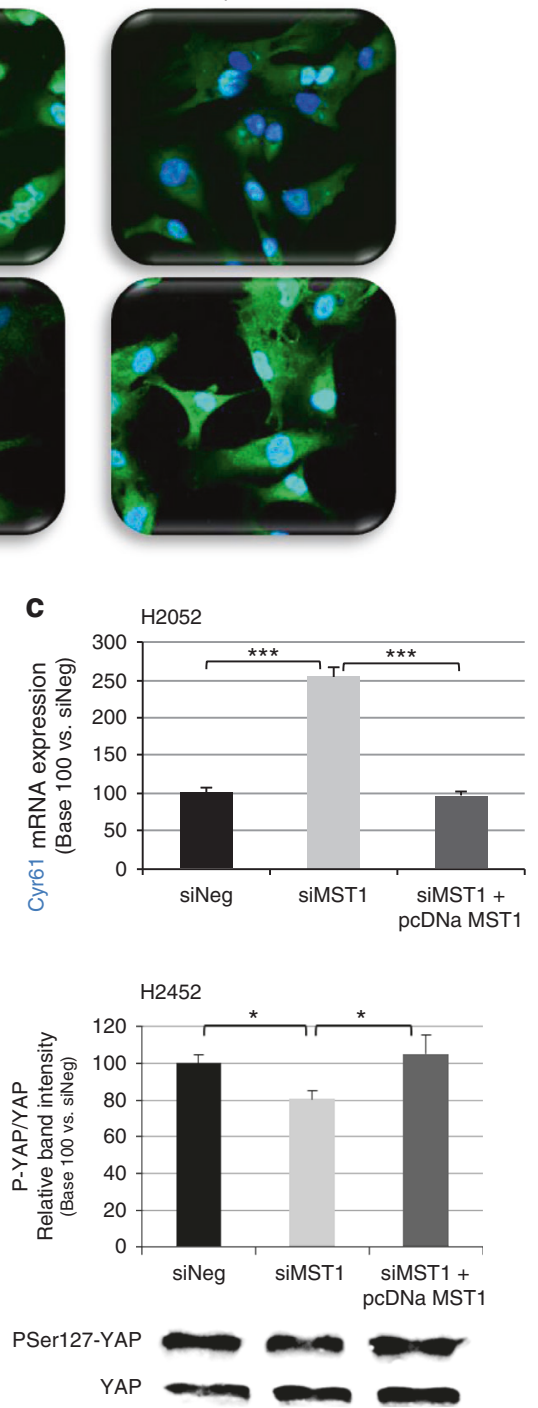

Fig. 5 MST1 depletion increases nuclear Yes-associated protein (YAP) but decreases nuclear transcriptional co-activator with PDZ-binding motif (TAZ). a-d MST0-211H, H2452, and H2052 cells were transfected with siNeg, siMST1, or siMST1+pcDNAMST1. Experiments performed $48 \mathrm{~h}$ after transfection. a YAP nuclear and cytoplasmic localisation assayed by immunofluorescence. YAP/TAZ activity by quantifying ANKDR1 expression in b H2452 or c H2052 cells. d Quantification of TAZ, YAP, and PSer127-YAP protein levels using glyceraldehyde-3-phosphate dehydrogenase (GAPDH) as internal control. For all histograms, error bars indicate the SEM of at least three independent experiments; ${ }^{*} p<$ $0.05,{ }^{* *} p<0.01$, and ${ }^{* * *} p<0.001$, using an analysis of variance (ANOVA) test followed by Dunnett's test 

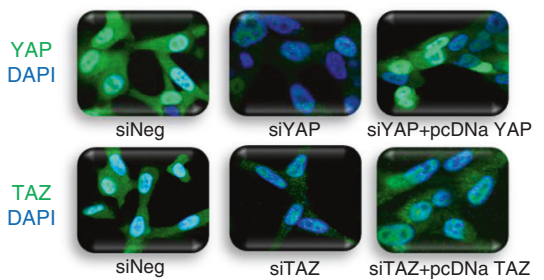

b

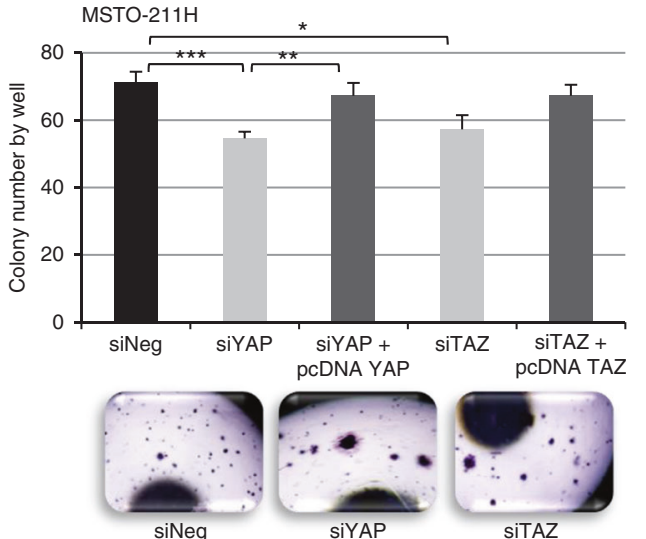

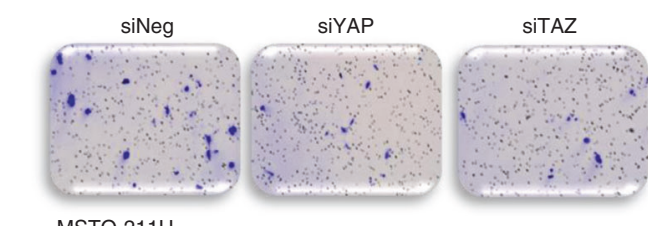
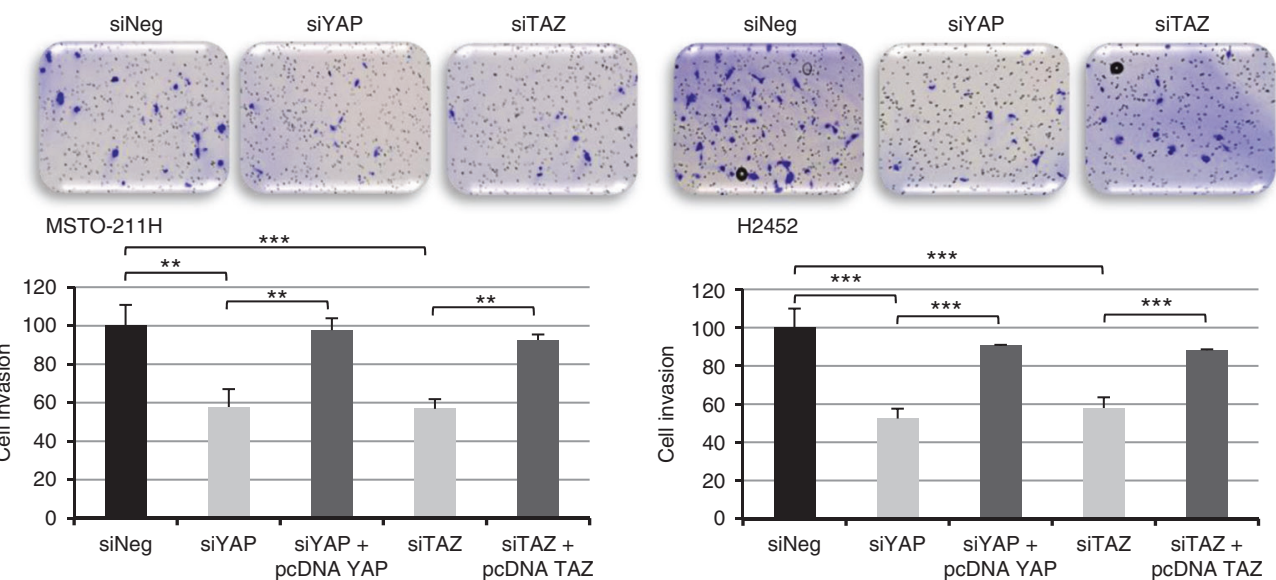

$\mathrm{H} 2452$

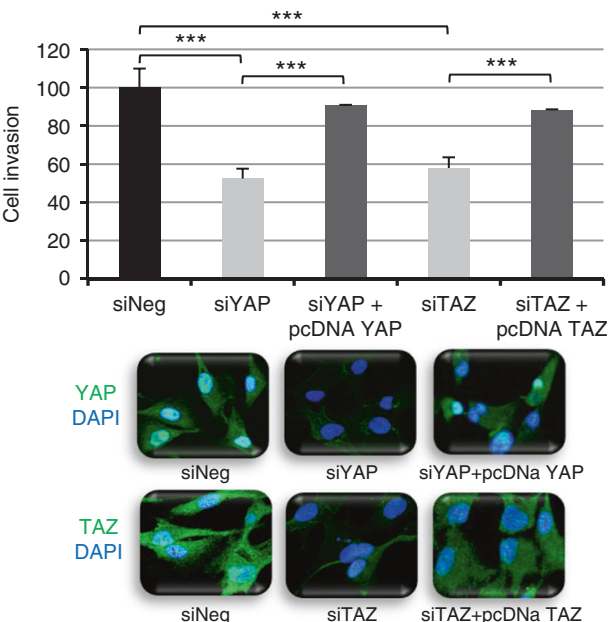

H2452

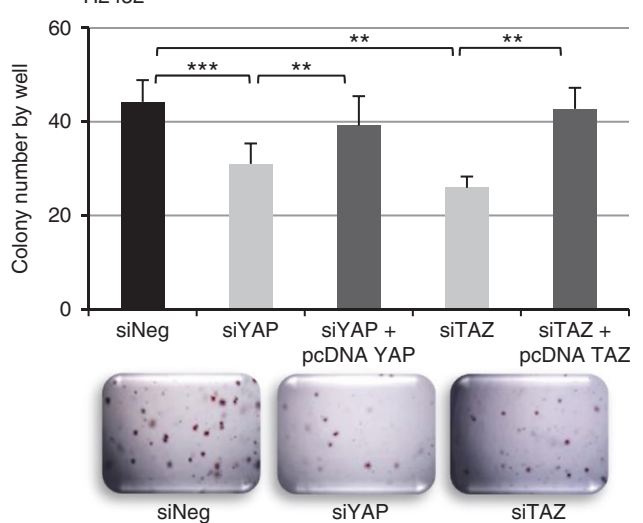

C

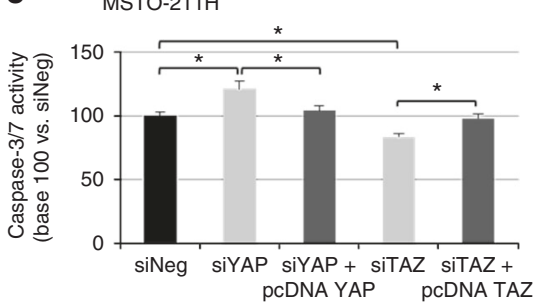

d MSTO-211H

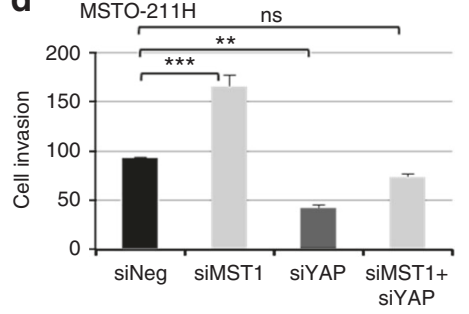

MSTO-211H
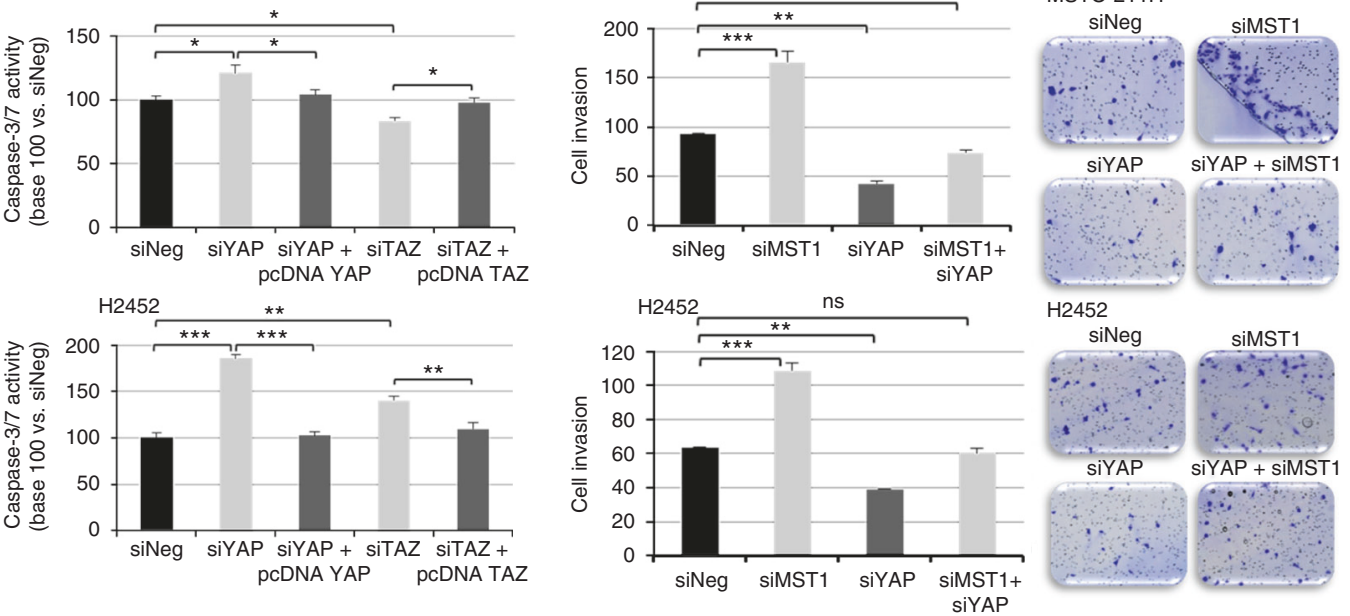

Fig. 6 Yes-associated protein (YAP) or transcriptional co-activator with PDZ-binding motif (TAZ) depletion decreases cell invasion and anchorage-independent growth while increasing apoptosis. a-d MSTO-211H and H2452 cells were transfected with siNeg, siYAP, siTAZ, and siMST1 in combination or not with pCDNA YAP or TAZ. Experiments performed $48 \mathrm{~h}$ after transfection. a Invasion capacity of transfected cells on BioCoat Matrigel Invasion Chamber; representative image of YAP and TAZ extinction shown under the histograms. $\mathbf{b}$ Quantification of colonies in soft agar after 21 days. Representative picture is provided above (a) or under (b) histograms. c Caspase 3/7 activity. d Invasion capacity of transfected cells on BioCoat Matrigel Invasion Chamber for $48 \mathrm{~h}$. For all histograms, error bars indicate the SEM of at least three independent experiments; ${ }^{*} p<0.05,{ }^{* *} p<0.01$, and ${ }^{* * *} p<0.001$, using an analysis of variance (ANOVA) test followed by Dunnett's test 
MSTO-211H cells, adherent or anchorage-independent cell growth was suppressed, as was colony formation. Similarly, another team recently experimented with $\mathrm{H} 2452$ cells with wild-type LATS1, using YAP RNAi-mediated knockdown, demonstrating that such YAP depletion also inhibited the growth of H2452 mesothelial cells and significantly suppressed Hippo pathway transcriptional activity, with decreased invasion, tumorosphere formation, and impaired stem cell self-renewal capacity. ${ }^{38}$ These data, along with our own findings, support the putative therapeutic potential of YAP inhibition in MPM.

Routinely performing MST1 methylation assay could thus help identify a subset of patients with poorer prognosis who could derive benefit from therapies targeting YAP, for instance by impairing the interaction of YAP with its transcriptional partners (e.g., TEAD 1-4 proteins). Our results, obtained in this homogeneous large series of MPM patients, all treated in a large phase 3 trial, confirm that targeting YAP in MPM represents a new and attractive therapeutic intervention in this disease, by targeting the actual features of MPM aggressiveness, responsible for unsatisfactory survival even under modern triplet regimens such as that used in the experimental arm of the MAPS trial, associating chemotherapy with the anti-angiogenesis drug bevacizumab.

\section{ACKNOWLEDGEMENTS}

The authors thank the CMABio3 platform and Dr. N. Richard of the Department of Genetics of the University Hospital of Caen for the molecular characterisation of the mesothelial cell line used in this work. The Bio-MAPS study was sponsored by the Intergroupe Francophone de Cancérologie Thoracique (IFCT), funded by an unrestricted research grant from ROCHE-France, research grants from ROCHE-France (2013) and from the Fond de Recherche en Santé Respiratoire (FRSR, 2012) to G.L., a research grant "APRI" from Caen University Hospital for S.B. (2014), and a research grant from the Normandie League against cancer for G.Z. (2012).

\section{AUTHOR CONTRIBUTIONS}

G.L and G.Z. conceived and designed the experiments. G.L., E.M., and S.B. performed experiments. V.H. provided technical assistance for in vivo experiments. C.D. validated the MPM diagnostic of each patient from the MAPS trial. E.B., A.S., J.M., J.M., L.G., C.A.V., D.M-S., O.M., R.M., I.M., and V.G. participated to sample collection. A.L. and F.M. performed data collection. A.L., F.M. and C.C. performed data analysis. G.L., G.Z., A.L., and C.C. performed data interpretation. E.M., G.L. and G.Z. wrote the manuscript with the help of all the authors. All authors approved the final manuscript.

\section{ADDITIONAL INFORMATION}

Supplementary information is available for this paper at https://doi.org/10.1038/ s41416-019-0379-8.

Competing interests: The authors declare no competing interests.

Data availability: All data are stored at the IFCT centre and can be made available upon request.

Ethics approval and consent to participate: Specific informed consent was obtained for the biological studies (Bio-MAPS), and the trial was approved by the appropriate ethics committee (CPP Ref 2007-20 Nord-Ouest III, France).

Note: This work is published under the standard license to publish agreement. After 12 months the work will become freely available and the license terms will switch to a Creative Commons Attribution 4.0 International (CC BY 4.0).

Publisher's note: Springer Nature remains neutral with regard to jurisdictional claims in published maps and institutional affiliations.

\section{REFERENCES}

1. Wagner, J. C., Sleggs, C. A. \& Marchand, P. Diffuse pleural mesothelioma and asbestos exposure in the North Western Cape Province. Br. J. Ind. Med. 17, 260-271 (1960)
2. Zalcman, G. et al. Bevacizumab for newly diagnosed pleural mesothelioma in the Mesothelioma Avastin Cisplatin Pemetrexed Study (MAPS): a randomised, controlled, open-label, phase 3 trial. Lancet 387, 1405-1414 (2016).

3. Bueno, R. et al. Comprehensive genomic analysis of malignant pleural mesothelioma identifies recurrent mutations, gene fusions and splicing alterations. Nat. Genet. 48, 407-416 (2016).

4. Meng, Z., Moroishi, T. \& Guan, K.-L. Mechanisms of Hippo pathway regulation. Genes Dev. 30, 1-17 (2016).

5. Qin, F., Tian, J., Zhou, D. \& Chen, L. Mst1 and Mst2 kinases: regulations and diseases. Cell Biosci. 3, 31 (2013).

6. Bitra, A., Sistla, S., Mariam, J., Malvi, H. \& Anand, R. Rassf proteins as modulators of Mst1 kinase activity. Sci. Rep. 7, 45020 (2017).

7. Guo, C., Zhang, X. \& Pfeifer, G. P. The tumour suppressor RASSF1A prevents dephosphorylation of the mammalian STE20-like kinases MST1 and MST2. J. Biol. Chem. 286, 6253-6261 (2011).

8. Servas, C. et al. The mammalian STE20-like kinase 1 (MST1) is a substrate for the apoptosis inhibiting protein kinase CK2. Cell. Signal. 36, 163-175 (2017).

9. Chiba, S., Ikeda, M., Katsunuma, K., Ohashi, K. \& Mizuno, K. MST2- and Furrymediated activation of NDR1 kinase is critical for precise alignment of mitotic chromosomes. Curr. Biol. 19, 675-681 (2009).

10. Guo, C. et al. RASSF1A is part of a complex similar to the Drosophila Hippo/ Salvador/Lats tumour-suppressor network. Curr. Biol. 17, 700-705 (2007).

11. Hergovich, A. et al. The MST1 and hMOB1 tumour suppressors control human centrosome duplication by regulating NDR kinase phosphorylation. Curr. Biol.19, 1692-1702 (2009).

12. Praskova, M., Xia, F. \& Avruch, J. MOBKL1A/MOBKL1B phosphorylation by MST1 and MST2 inhibits cell proliferation. Curr. Biol. 18, 311-321 (2008).

13. Robertson, A. et al. Genetic ablation of the mammalian sterile-20 like kinase 1 (Mst1) improves cell reprogramming efficiency and increases induced pluripotent stem cell proliferation and survival. Stem Cell Res. 20, 42-49 (2017).

14. Katagiri, K. et al. Mst1 controls lymphocyte trafficking and interstitial motility within lymph nodes. EMBO J. 28, 1319-1331 (2009).

15. Liang, $Y$. et al. Long-term in vivo single-cell tracking reveals the switch of migration patterns in adult-born juxtaglomerular cells of the mouse olfactory bulb. Cell Res. 26, 805-821 (2016).

16. $\mathrm{Xu}, \mathrm{X}$. et al. Mst1 kinase regulates the actin-bundling protein L-plastin to promote T cell migration. J. Immunol. 197, 1683-1691 (2016).

17. Fischer, J. R. et al. Promoter methylation of RASSF1A, RARbeta and DAPK predict poor prognosis of patients with malignant mesothelioma. Lung Cancer 54, 109-116 (2006).

18. Toyooka, S. et al. Progressive aberrant methylation of the RASSF1A gene in simian virus 40 infected human mesothelial cells. Oncogene 21, 4340-4344 (2002).

19. de Fraipont, F. et al. An apoptosis methylation prognostic signature for early lung cancer in the IFCT-0002 trial. Clin. Cancer Res. 18, 2976-2986 (2012).

20. Seidel, C. et al. Frequent hypermethylation of MST1 and MST2 in soft tissue sarcoma. Mol. Carcinog. 46, 865-871 (2007).

21. Levallet, G. et al. Plasma cell membrane localization of c-MET predicts longer survival in patients with malignant mesothelioma: a series of 157 cases from the MESOPATH Group. J. Thorac. Oncol. 7, 599-606 (2012).

22. Dubois, F. et al. RASSF1A suppresses the invasion and metastatic potential of human non-small cell lung cancer cells by inhibiting YAP activation through the GEF-H1/RhoB pathway. Cancer Res. 76, 1627-1640 (2016).

23. Miyanaga, A. et al. Hippo pathway gene mutations in malignant mesothelioma: revealed by RNA and targeted exon sequencing. J. Thorac. Oncol. 10, 844-851 (2015).

24. Woodard, G. A., Yang, Y.-L., You, L. \& Jablons, D. M. Drug development against the hippo pathway in mesothelioma. Transl. Lung Cancer Res. 6, 335-342 (2017).

25. Steinmann, K., Sandner, A., Schagdarsurengin, U. \& Dammann, R. H. Frequent promoter hypermethylation of tumour-related genes in head and neck squamous cell carcinoma. Oncol. Rep. 22, 1519-1526 (2009).

26. Fukuhara, T. et al. Hypermethylation of MST1 in IgG4-related autoimmune pancreatitis and rheumatoid arthritis. Biochem. Biophys. Res. Commun. 463, 968-974 (2015).

27. Sekido, Y. Inactivation of Merlin in malignant mesothelioma cells and the Hippo signaling cascade dysregulation: Merlin inactivation in mesothelioma. Pathol. Int. 61, 331-344 (2011).

28. Tranchant, R. et al. Co-occurring mutations of tumour suppressor genes, LATS2 and NF2, in malignant pleural mesothelioma. Clin. Cancer Res. 23, 3191-3202 (2017).

29. Lin, X. et al. Prognostic significance of mammalian sterile 20-like kinase 1 in breast cancer. Tumour Biol. 34, 3239-3243 (2013).

30. Sohn, B. H. et al. Inactivation of Hippo pathway is significantly associated with poor prognosis in hepatocellular carcinoma. Clin. Cancer Res. 22, 1256-1264 (2016). 
31. $\mathrm{Yu}, \mathrm{J}$. Identification of MST1 as a potential early detection biomarker for colorectal cancer through a proteomic approach. Sci. Rep. 7, 14265 (2017).

32. Attarha, S., Andersson, S., Mints, M. \& Souchelnytskyi, S. Mammalian sterile-like 1 kinase inhibits TGF $\beta$ and EGF-dependent regulation of invasiveness, migration and proliferation of HEC-1-A endometrial cancer cells. Int. J. Oncol. 45, 853-860 (2014).

33. Fallahi, E., O'Driscoll, N. \& Matallanas, D. The MST/Hippo pathway and cell death: a non-canonical affair. Genes 7, 28 (2016).

34. Galan, J. A. \& Avruch, J. MST1/MST2 protein kinases: regulation and physiologic roles. Biochemistry 55, 5507-5519 (2016)

35. Oh, $\mathrm{H}$. J. et al. Role of the tumour suppressor RASSF1A in Mst1-mediated apoptosis. Cancer Res. 66, 2562-2569 (2006)
36. Xu, C., Liu, C., Huang, W., Tu, S. \& Wan, F. Effect of Mst1 overexpression on the growth of human hepatocellular carcinoma HepG2 cells and the sensitivity to cisplatin in vitro. Acta Biochim. Biophys. Sin. 45, 268-279 (2013).

37. Xu, C. M. et al. Mst1 overexpression inhibited the growth of human non-small cell lung cancer in vitro and in vivo. Cancer Gene Ther. 20, 453-460 (2013).

38. Zhang, W.-Q. et al. Targeting YAP in malignant pleural mesothelioma. J. Cell. Mol. Med. 21, 2663-2676 (2017).

39. Wang, C. et al. Verteporfin inhibits YAP function through up-regulating 14-3-30 sequestering YAP in the cytoplasm. Am. J. Cancer Res. 6, 27-37 (2015). 